\title{
RF Field Build-Up inside a Manned Space Vehicle Using Novel Ray-Tracing Algorithm
}

\author{
Balamati Choudhury, Hema Singh, and R. M. Jha \\ Centre for Electromagnetics, CSIR-National Aerospace Laboratories, Bangalore 560017, India \\ Correspondence should be addressed to Balamati Choudhury; balamati@nal.res.in
}

Received 25 February 2013; Accepted 3 October 2013

Academic Editor: Linda L. Vahala

Copyright ( 2013 Balamati Choudhury et al. This is an open access article distributed under the Creative Commons Attribution License, which permits unrestricted use, distribution, and reproduction in any medium, provided the original work is properly cited.

\begin{abstract}
The radio-frequency (RF) field mapping and its analysis inside a space vehicle cabin, although of immense importance, represent a complex problem due to their inherent concavity. Further hybrid surface modeling required for such concave enclosures leads to ray proliferation, thereby making the problem computationally intractable. In this paper, space vehicle is modeled as a doublecurvatured general paraboloid of revolution (GPOR) frustum, whose aft section is matched to an end-capped right circular cylinder. A $3 \mathrm{D}$ ray-tracing package is developed which involves a uniform ray-launching scheme, an intelligent scheme for ray bunching, and an adaptive reception algorithm for obtaining ray-path details inside the concave space vehicle. Due to nonavailability of image method for concave curvatured surfaces, the proposed ray-tracing method is validated with respect to the RF field build-up inside a closed lossy cuboid using image method. The RF field build-up within the space vehicle is determined using the details of ray paths and the material parameters. The results for RF field build-up inside a metal-backed dielectric space vehicle are compared with those of highly metallic one for parallel and perpendicular polarizations. The convergence of RF field within the vehicle is analyzed with respect to the propagation time and the number of bounces a ray undergoes before reaching the receiving point.
\end{abstract}

\section{Introduction}

In the fore section of any space vehicle, astronauts work in the presence of multiple radiating sources. This makes the astronaut cabin of space vehicle an important indoor environment which necessitates RF field mapping. The space vehicle cabin is essentially a concave structure. Over the last decades, ray tracing has been employed for site-specific indoor propagation models [1-3], and it has been shown that multiple reflections are dominant for the RF field build-up within the cavity compared to the phenomenon of diffraction [4]. Although ray tracing has been used earlier for electromagnetic (EM) analysis of aircraft cabin-like enclosures, a closer scrutiny reveals that these predominantly employ measurements to merely fit their predictions or to validate their empirical models [5-7].

For EM environment analysis within the space shuttle interior (payload bay area), attempts have been made earlier to overcome the computational complexity by approximating the curved surfaces with large planar faceted plates [8].
However, this leads to a completely different ray solution set, which may not necessarily approximate to the case of curvatured surfaces under consideration. Further there are sections of space vehicle, which cannot be approximated by single-curvature surfaces (e.g., circular and elliptic cylinders) and necessitate modeling with double-curvatured surfaces (e.g., spherical, paraboloidal, and ellipsoidal sections). EM analysis for convex structures, including double-curvatured structures, has been done using high-frequency methods along with analytical ray-tracing technique such as the Geodesic Constant Method (GCM) [9]. But this is restricted only to the external geometries, that is, the convex part of such aerospace structures.

The ray tracing becomes extremely cumbersome in the important applications of crevices and concavities within an enclosure (such as space vehicles, aircraft engine, cockpit, and passenger cabins) due to ray proliferation, arising from multiple reflections, transmission, and diffraction. In fact the only route to ray tracing available in such cases is ray casting [7], which improves the prediction only when the spatial rays are 


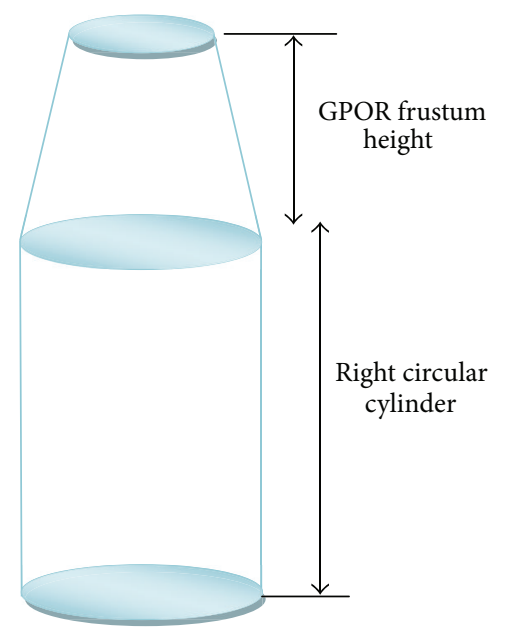

Figure 1: Schematic of the space vehicle.

increasingly dense, leading to computational intractability. Hence, a feasible ray-tracing method is required to generate ray-path data for practical applications.

In the present work, a novel refined ray-tracing method is developed for RF field computation inside a space vehicle including that for the microwave frequencies in the $\mathrm{GHz}$ region. A comprehensive $3 \mathrm{D}$ ray-tracing package is developed in conjunction with analytical surface modeling to generate the ray-path data inside a typical space vehicle. The proposed ray-tracing method is validated with respect to the RF field build-up inside a closed lossy cuboid using the image method. This is because the image method is known to be valid for planar surfaces, whereas the concave curvatured surfaces like space vehicle or aircraft in-cabin have mostly nonplanar surfaces. The space vehicle is modeled as a hybrid of right circular cylinder and a general paraboloid of revolution (GPOR) frustum. The aft section is modelled by an endcapped right circular cylinder (Figure 1). The dimensions of a currently used space vehicle are considered for the RF simulations [10]. Test rays are launched from a radiating source located inside the space vehicle and are allowed to propagate inside the space vehicle. The reflection points at each surface and the corresponding reflected rays are obtained using the parametric equation of the surface and the surface normal [9] at each incident point. As the surface parameters of the modeled space vehicle are defined through analytical equations, the ray-path computation time reduced significantly.

The cumulative ray-path data up to $N$ th bounce including the direct ray is used for the estimation of RF field inside a space vehicle. It includes the details of each ray path traversed within the space vehicle before reaching the receiving point. Moreover the constitutive parameters of the space vehicle material are taken into account in calculating the total reflected power at the receiving point. It is well known that, unlike the convex surfaces, the RF field mapping inside a concave structure is an extremely complex problem dominated by the proliferation of reflected ray solutions. Hence in this paper, an empty space vehicle cabin is considered to analyze the problem of concavity. The transmitting and the receiving antennas are taken as a directional antenna (halfwavelength dipole). The RF field build-up at the receiver placed within a manned space vehicle is reported for both the perpendicular and parallel polarizations. The resultant field build-up at the receiving point is obtained by a coherent summation of the fields associated with each ray reaching the receiver after undergoing multiple bounces from the walls of the space vehicle. The results for a metallic space vehicle are compared with that of metal-backed dielectric space vehicle. The convergence of the RF field build-up is studied with respect to the propagation time and the number of reflections a ray undergoes before reaching the receiving point.

\section{Surface Modeling of a Space Vehicle}

Hybrid quadratics such as general paraboloid of revolution (GPOR) frustum and finite right circular cylinder are sufficient enough to model a typical space vehicle for RF analysis. In analytical surface modeling, the surfaces are defined by parametric equations and the shaping parameters of the corresponding surfaces are calculated towards perfect matching.

2.1. Geometry of Space Vehicle. The schematic of the space vehicle is shown in Figure 1. The vehicle is modeled as a double-curvatured general paraboloid of revolution (GPOR) frustum, whose aft section is matched to an end-capped right circular cylinder. To describe the analytical modeling, the dimensions of a typical space vehicle considered [10] are taken as follows:

$$
\begin{aligned}
& \text { length of the cylindrical body: } 7.50 \mathrm{~m} \text {; } \\
& \text { right circular cylinder diameter: } 4.50 \mathrm{~m} \text {; } \\
& \text { matched GPOR diameter: } 4.50 \mathrm{~m} \text {; }
\end{aligned}
$$$$
\text { height of GPOR frustum: } 5.00 \mathrm{~m} \text {. }
$$

\subsection{Calculation of Shaping Parameters in Hybrid Structure.} The parametric equation of right circular cylinder is given by [9]

$$
x=\rho_{\text {cyl }} \cos \phi, \quad y=\rho_{\text {cyl }} \sin \phi, \quad v_{1} \leq z \leq v_{2},
$$

where $\rho_{\text {cyl }}$ is the radius of the right circular cylinder, $\phi$ is its azimuth angle that varies from 0 to $360^{\circ}$, and $\left(v_{2}-\right.$ $v_{1}$ ) represents the finite length of the cylinder. To model the hybrid structure, one needs to determine the shaping parameters of the structure. The parametric equation of a GPOR is given by [11]

$$
x=a u \cos \phi, \quad y=a u \sin \phi, \quad z=-u^{2},
$$

where $\phi$ is now the azimuth angle of GPOR varying from 0 to $360^{\circ}, u$ is the GPOR basis parameter, and $a$ is the shaping parameter that represents the sharpness/flatness of the GPOR. 


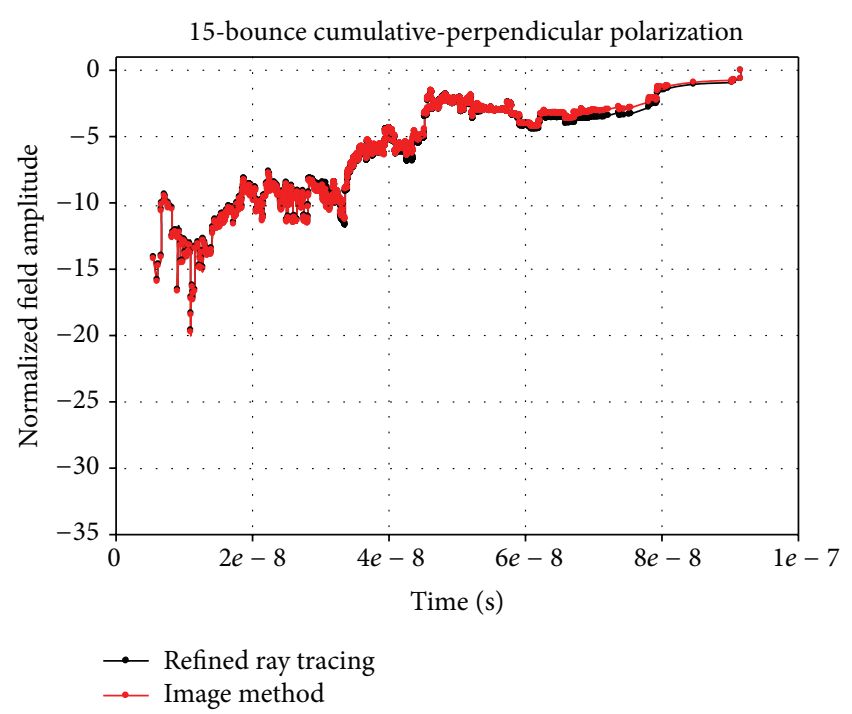

FIGURE 2: RF field build-up at the receiver inside a lossy cuboid using image method and refined ray tracing method.

\section{Propagation inside a Space Vehicle}

A novel refined ray-tracing algorithm is developed for ray propagation analysis inside the concavities. The developed algorithm is validated with respect to a lossy cuboid using image method as image method is not available for concave surfaces. The proposed refined ray-tracing algorithm along with the RF field validation is given below.

3.1. Validation of Refined Ray-Tracing Algorithm. As image method is simply not available for curved concavities such as the space vehicle, the RF field due to the rays obtained by the existing image method inside a lossy cuboid $(2.70 \mathrm{~m}$ $\times 2.38 \mathrm{~m} \times 2.40 \mathrm{~m}$ ) and those obtained by the refined raytracing method developed in-house are compared. The RF field build-up analysis is carried at $2.4 \mathrm{GHz}$. The source is kept at origin $(0,0,0)$. The receiver is at $(0.9,1.85,1.1)$. The cumulative ray paths up to 15 bounces are considered for the simulation studies. The constitutive parameters of the lossy walls of the cuboid are taken into account while estimating the net field build-up. Figure 2 presents the RF field buildup at the receiving point within the lossy cuboid $\left(\varepsilon_{r}=\right.$ $10.74-j 2.01, d=4 \mathrm{~mm}$ ) for perpendicular polarization. It may be observed that the refined ray-tracing algorithm has excellent agreement with image method in providing ray-path descriptions for RF field build-up within the closed cavity.

3.2. Refined Ray Tracing inside the Space Vehicle. As described above, a closed analytically modeled hybrid space vehicle is considered for ray-propagation analysis and the transmitter and the receiver are located inside the space vehicle without loss of generality at $S(0.5,0.9,-15.5)$ and $R(0.5,-0.4,-6.0)$. Figure 3 illustrates the schematic of the same.

Initially a uniform ray-launching scheme [12] is used from the isotropic source (transmitter), which is subsequently

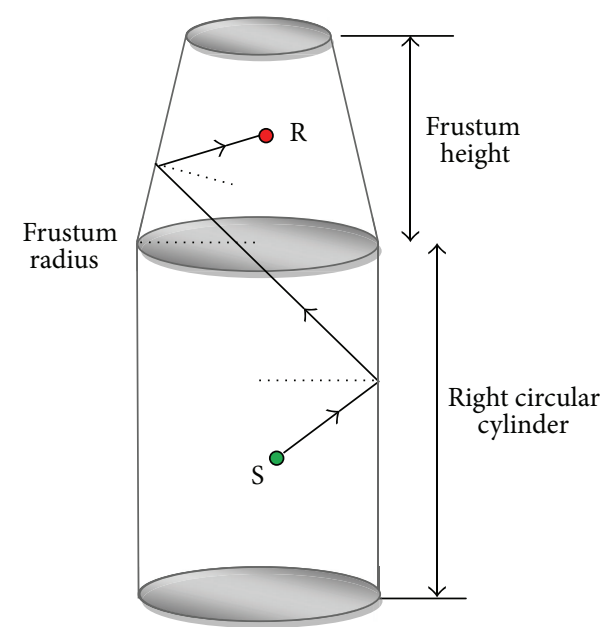

FIGURE 3: Schematic of the ray propagation inside a space vehicle.

adapted for convergence of ray solutions at the receiver. Each ray is uniquely defined by its $(\theta, \phi)$ values. The rays are then allowed to propagate inside the cabin. The first intersection point is determined by the intersection formula between a line and the surface of the corresponding hybrid structure [13].

As the hybrid structure has four different quadratic surfaces at different heights, initial intersection point is checked with respect to $z$-coordinate and the surface equation is adopted for calculation of first incident point. The unit surface normal vector (Figure 4) at the first intersection point is calculated using the following equations with respect to the surface [9].

The unit surface normal equation of a second degree quadric patch is given by

$$
\widehat{N}=x_{N} \widehat{i}+y_{N} \hat{j}+z_{N} \widehat{k}
$$

which for a right circular cylinder is expressed as

$$
x_{N}=\cos \phi, \quad y_{N} \sin \phi, \quad z_{N}=0,
$$

while for the GPOR, it is given by

$$
x_{N}=\frac{2 u \cos \phi}{\sqrt{a^{2}+4 u^{2}}} \quad y_{N}=\frac{2 u \sin \phi}{\sqrt{a^{2}+4 u^{2}}} \quad z_{N}=\frac{a}{\sqrt{a^{2}+4 u^{2}}} .
$$

The unit surface normal corresponding to the above four constituent surfaces of the hybrid structure is shown in Figure 4.

Once the surface normal at the point (where the ray is incident) is determined, the reflected ray is obtained by employing the Snell law of reflection. This process is repeated for the subsequent bounces.

An adaptive reception sphere is considered to capture the rays. The rays that reach the reception sphere are considered as the required set of rays for the RF field build-up inside the cabin. Further, an intelligent ray-bunching scheme is used to avoid duplication of ray solutions. 
TABLE 1: Rays reaching receiver cumulatively up to $N$-bounce and the execution time (4126183 rays are launched) in Intel Core Duo CPU, $3 \mathrm{GHz}$, and $4 \mathrm{~GB}$ RAM.

\begin{tabular}{lcc}
\hline$N$-Bounce & $\begin{array}{c}\text { Number of rays } \\
\text { received }\end{array}$ & $\begin{array}{c}\text { Program execution } \\
\text { time }\end{array}$ \\
\hline 1 bounce & 4 & $25 \mathrm{sec}$ \\
5 bounces & 120 & $1.55 \mathrm{~min}$ \\
10 bounces & 538 & $6.67 \mathrm{~min}$ \\
15 bounces & 893 & $11.00 \mathrm{~min}$ \\
20 bounces & 1082 & $17.56 \mathrm{~min}$ \\
25 bounces & 1205 & $24.35 \mathrm{~min}$ \\
30 bounces & 1279 & $29.45 \mathrm{~min}$ \\
35 bounces & 1318 & $34.12 \mathrm{~min}$ \\
40 bounces & 1345 & $42.35 \mathrm{~min}$ \\
\hline
\end{tabular}

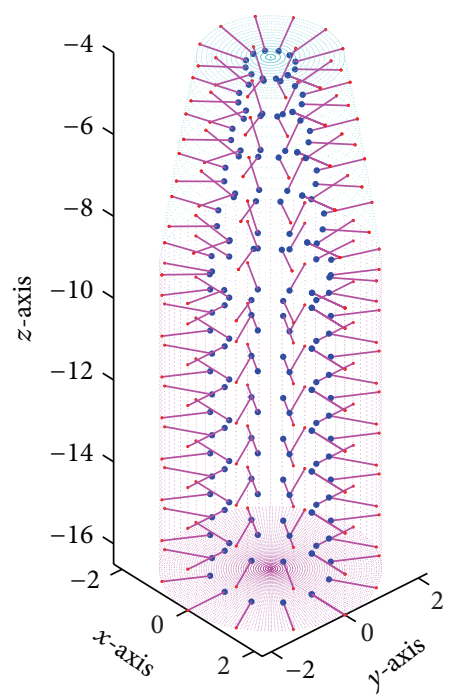

FIGURE 4: The unit surface normal vector representation of GPOR frustum and right circular cylinder.

3.3. Results and Visualization of Ray Path. The ray-path propagation characteristics inside the modeled space vehicle are checked for conditions of reflection, and the ray-path data up to 40 bounces (cumulative) are generated. An angular separation of $0.1^{\circ}$ is considered. It is observed that besides the direct ray only 66 rays reach the reception sphere cumulatively, up to 4 bounces. The ray-path visualization of these 66 rays is shown in Figure 5.

The corresponding number of rays converging on to the receiver with respect to $N$-bounce and the program execution time are given in Table 1.

It is pointed out that for the RF field mapping application under consideration, as discussed in the next section, it is sufficient to consider the ray-path data up to 40 bounces.

\section{RF Field Build-Up inside the Space Vehicle}

RF field mapping problem is formulated in three steps, namely, (i) analytical surface modeling of the space vehicle

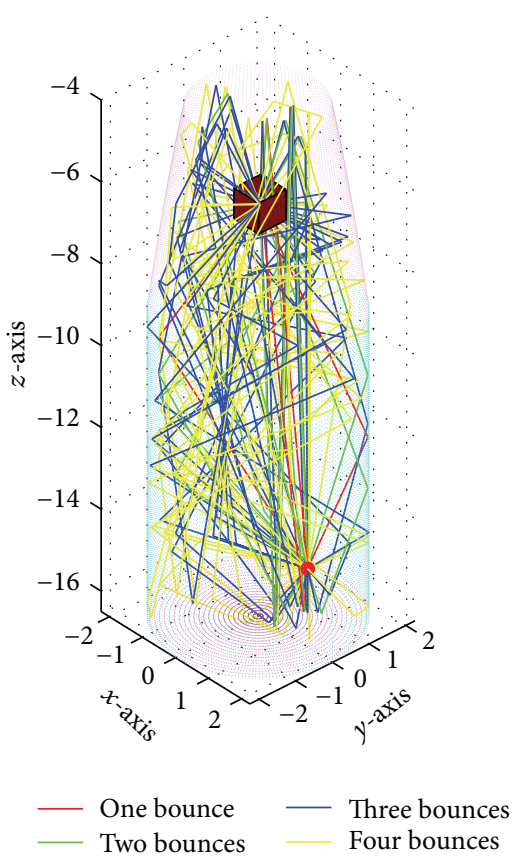

FIGURE 5: Ray-path visualization of cumulative rays up to four bounces inside the space vehicle.

enclosure and (ii) ray-path data generation within the enclosure using a refined ray-tracing algorithm, followed by (iii) frequency and polarization dependent EM field computation for the RF field mapping inside the space vehicle.

The RF field estimation within an enclosure needs the details of ray paths, reflection points, and radiation pattern of the transmitting and receiving antennas. In this paper, the ray-path details and the coordinates of reflection point(s) are obtained using a novel adaptive three-dimensional raytracing procedure, explained in the previous section. When multiple bounces and numerous ray paths are considered, the ray tracing becomes computationally intense and complicated. However, for an arbitrary concave environment, volumetric ray tracing still offers an efficient approach for RF simulation.

The resultant electric field at a receiving point $P$ within the enclosure [14] is given by

$$
E(P)=\sum_{i} E_{i}(P)=\sum_{i} E_{o} F_{t i} F_{r i}\left\{\prod_{l} R_{l}\right\} e^{-j k d} L_{i}(d),
$$

where $E_{i}(P)$ is the electric field due to the $i$ th ray at the receiver $P$. This ray experiences a finite number of reflections during its path from the source to the receiver. The number of the reflections a ray will undergo depends on the angle at which the ray is launched from the source.

The source is taken as a point source. The rays launched within the cavity are weighted according to the radiation pattern of the transmitting antenna. Similarly at the receiver, the rays are weighted in accordance with the radiation 

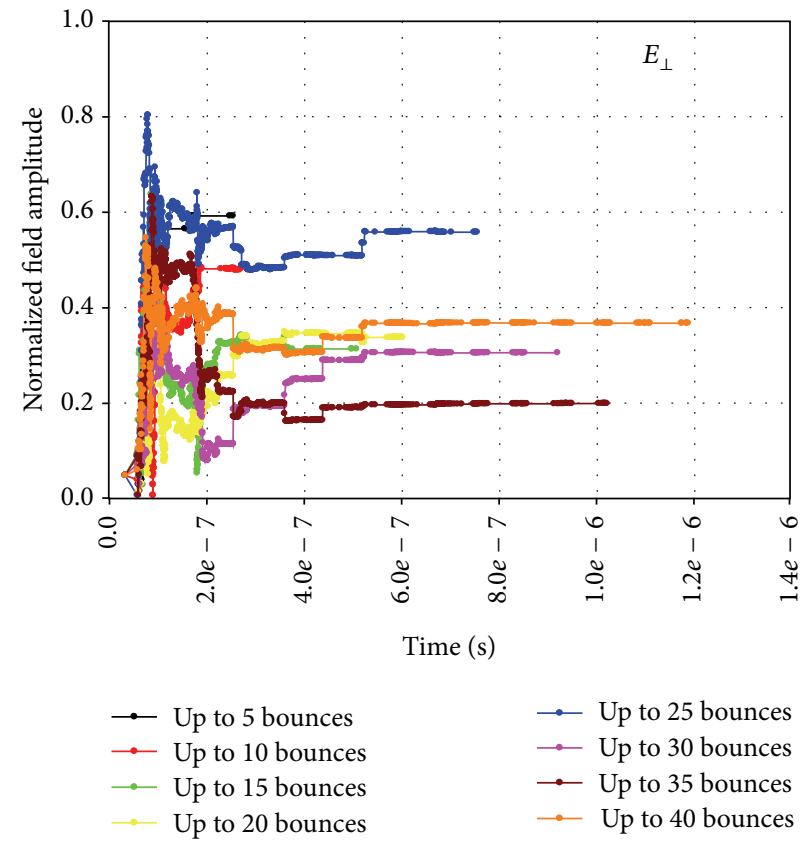

(a)
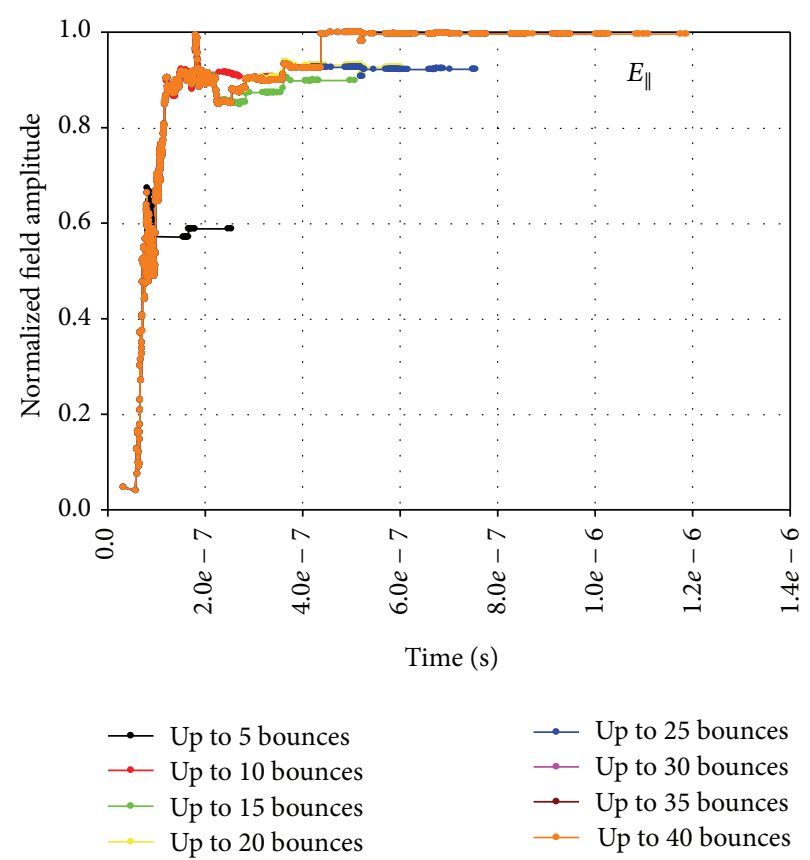

(b)

FIGURE 6: (a) RF field build-up inside a metallic space vehicle. Frequency $=6 \mathrm{GHz} ; \sigma=1.8 \times 10^{5} \mathrm{~S} / \mathrm{m}$, polarization: perpendicular. Since the walls of space vehicle are metallic, convergence has not been achieved. (b) RF field build-up inside a metallic space vehicle. Frequency $=$ $6 \mathrm{GHz} ; \sigma=1.8 \times 10^{5} \mathrm{~S} / \mathrm{m}$, polarization: parallel. Since the walls are metallic, convergence has not been achieved.

pattern of the receiving antenna. The time-independent field expressed in (4) is the coherent sum of field contributions of individual rays reaching the receiver. The phase of each ray (reaching the receiving point at different instants of time) is adjusted accordingly. $E_{o}$ is the initial field value at the source and $F_{t i}$ and $F_{r i}$ are the corresponding field values intercepted in the direction of ray. These field values are based on the radiation patterns of transmitting and receiving antennas, respectively. $R_{l}$ is the reflection coefficient of the $l$ th interface/wall [15-17], $d$ is the total ray-path length, $k$ is the wavenumber, and $L_{i}(d)$ is the path loss [14]. The dependence of the reflection coefficient on the material properties, namely, permittivity, conductivity, thickness, and the angle of incidence, is appropriately incorporated.

\section{Results and Discussion}

The RF simulations are carried out for both a metallic $(\sigma=$ $1.8 \times 10^{5} \mathrm{~S} / \mathrm{m}$ ) space vehicle and a metal-backed dielectric $\left(\varepsilon_{r}=10-j 5 ; d=5 \mathrm{~mm}\right)$ space vehicle. The transmitting and receiving antennas are assumed to be directional (as halfwave dipole).

The launching of rays within an enclosure can be done efficiently for any angular separation. However, if the angular separation between the adjacent launched rays is too large, fewer test rays are launched and the RF field does not converge. This implies the requirement of launching the test rays at increasingly smaller angles, which at the other extreme is beset with the problem of computational intractability. It has been observed that a trade-off exists for the class of problems being analyzed here, optimally in the range of $0.1^{\circ}$ to $0.25^{\circ}$. In this study, the RF field build-up at the receiving point inside a space vehicle is obtained for the ray angular separation of $0.1^{\circ}$ launched within the space vehicle. The effect of the frequency of operation on the RF field build-up is also studied. Simulations are carried out for two distinct frequencies of 6 and $8 \mathrm{GHz}$ for both the parallel and perpendicular polarizations. It is pointed out that these distinct frequencies are commonly used in space and satellite communication applications.

First, the RF field build-up is estimated for the interior of a highly metallic space vehicle. The normalized RF field build-up at the receiving point inside the metallic space vehicle at $6 \mathrm{GHz}$ is shown in Figures 6(a) and 6(b) for the perpendicular and parallel polarization, respectively. The corresponding results at $8 \mathrm{GHz}$ are given in Figures 7 (a) and 7 (b), respectively. For a given ray bounce, the complete set of rays is characterized by distinct $t_{\min }$ and $t_{\max }$. Here, $t_{\min }$ refers to the minimum time of the (shortest) ray path for a given bounce. Likewise, $t_{\max }$ corresponds to the maximum time of the (longest) ray within the set of rays for the same number of bounces. It is pointed out that, for the preset number of bounces, $t_{\min }$ may be more than the propagation time of several rays of a lower bounce type and even than some of the rays of the higher bounce type. Hence one of the focuses of this study is to analyze the RF field convergence with respect to the cumulative bounce of the feasible ray paths (i.e., all the ray paths cumulatively up to a preset number of bounces).

In the case of perpendicular polarization (Figures 6(a) and $7(a)$ ), the field convergence requires a very large number of bounces, and hence the time axis ought to be very 


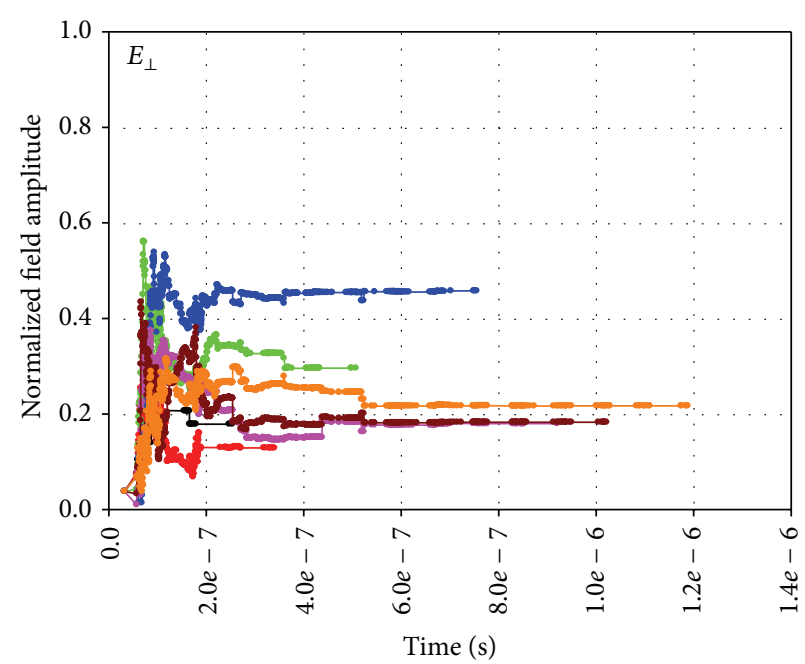

$\longrightarrow$ Up to 5 bounces
$\longrightarrow$ Up to 10 bounces
$\longrightarrow$ Up to 15 bounces
$\longrightarrow$ Up to 25 bounces

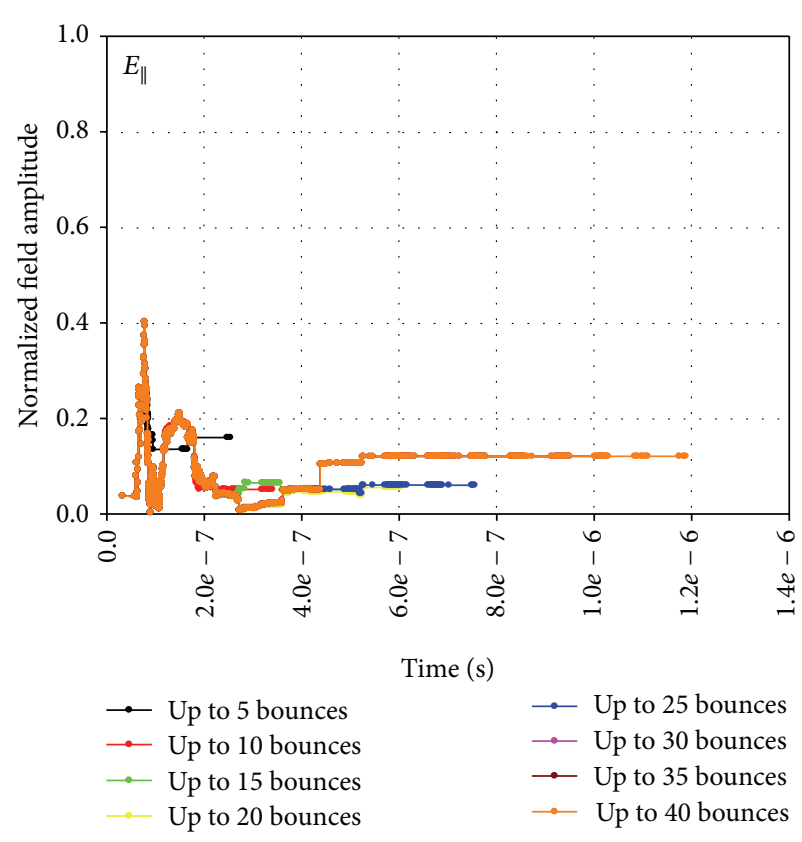

Time (s)

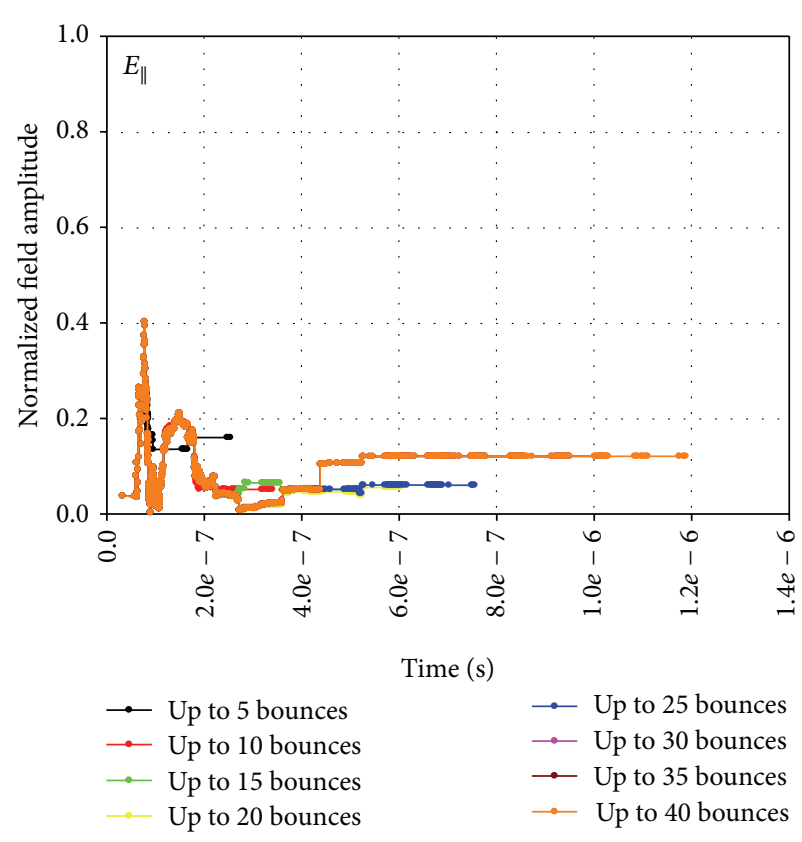

(b)

(a)

\begin{abstract}
—Up to 30 bounces
- Up to 35 bounces

-Up to 40 bounces
\end{abstract}

Figure 7: (a) RF field build-up inside a metallic space vehicle. Frequency $=8 \mathrm{GHz} ; \sigma=1.8 \times 10^{5} \mathrm{~S} / \mathrm{m}$, polarization: perpendicular. Since the walls are metallic, convergence has not been achieved. (b) RF field build-up inside a metallic space vehicle. Frequency $=8 \mathrm{GHz} ; \sigma=$ $1.8 \times 10^{5} \mathrm{~S} / \mathrm{m}$, polarization: parallel. Since the walls are metallic, convergence has not been achieved.

large. However, the time window considered till $1.2 \mu \mathrm{sec}$ is sufficient for practical applications as shown later. The results are reported till 40 bounces (cumulative), which converges within the ambit of computational tractability.

Since the walls of the space vehicle are considered to be highly metallic $\left(\sigma=1.8 \times 10^{5} \mathrm{~S} / \mathrm{m}\right)$, the rays do not suffer significant attenuation at each reflection before reaching the receiver. It can be observed from Figures 6(a) and 7(a) that the level of RF field build-up for perpendicular polarization tends to broadly increase with the number of bounces considered. This is ascribed to the fact that, as the number of bounces increases, the number of rays reaching the receiver also increases. These numerous rays reaching the receiver arrive from different directions and hence tend to substantially cancel leaving only a small net (positive or negative) RF field. This small net field contributes to the earlier threshold of the RF field build-up in the incremental sense, thereby leading to the extremely slow rate of convergence for the metallic case. This is on the expected lines since convergence has not been achieved within a highly metallic reverberation chamber-like environment [18].

In contrast, for the parallel polarization (Figures 6(b) and 7(b)), despite metallic walls, the RF field build-up converges rapidly. This is due to the fact that, for the parallel polarization, the direction of E-field vector changes continually [17] after successive (oblique-incidence) reflections. When the electric field associated with the multiple rays reaching the receiver is coherently superimposed, the fields with opposite phase tend to cancel each other, resulting in the rapid convergence of the total field build-up with respect to the number of bounces.
The amplitude of total RF field build-up at receiver, particularly initially, fluctuates with the number of bounces. This may be due to the fact that this study is emphasized with respect to the preset cumulative number of bounces, which is used as the cut-off that ignores all the higher-bounce ray paths. Although the higher bounces tend to result in increasingly diminished (and hence negligible) electric field contributions, their contribution during the initial RF buildup is not necessarily so, thereby leading to the observed RF fluctuations. However, with the increase in time, this error tends to cancel itself out.

A discerning reader may also notice that the total RF field build-up inside a metallic space vehicle is higher at $6 \mathrm{GHz}$ as compared to $8 \mathrm{GHz}$ for both the polarizations. This is explained by the fact that the geometric path-length solutions (between the source and receiver points) are independent of frequency. Thus compared to $8 \mathrm{GHz}$, each ray traverses fewer electrical wavelengths over the fixed geometric path at $6 \mathrm{GHz}$, leading to lesser attenuation and hence in general higher RF field build up values.

A metal-backed dielectric space vehicle is considered next. This dielectric in reality is highly absorbent in nature. Thus, a typical absorber permittivity is taken here, as $\varepsilon_{r}=$ $10-j 5 ; d=5 \mathrm{~mm}$. These constitutive parameters of the wall material are employed in the estimation of reflection coefficients [17] of each of the ray paths, as a function of polarization and all the successive angles of incidence. Thus, the contribution of each ray path reaching the receiver within the space vehicle can be coherently summed up after adjusting them in phase. 


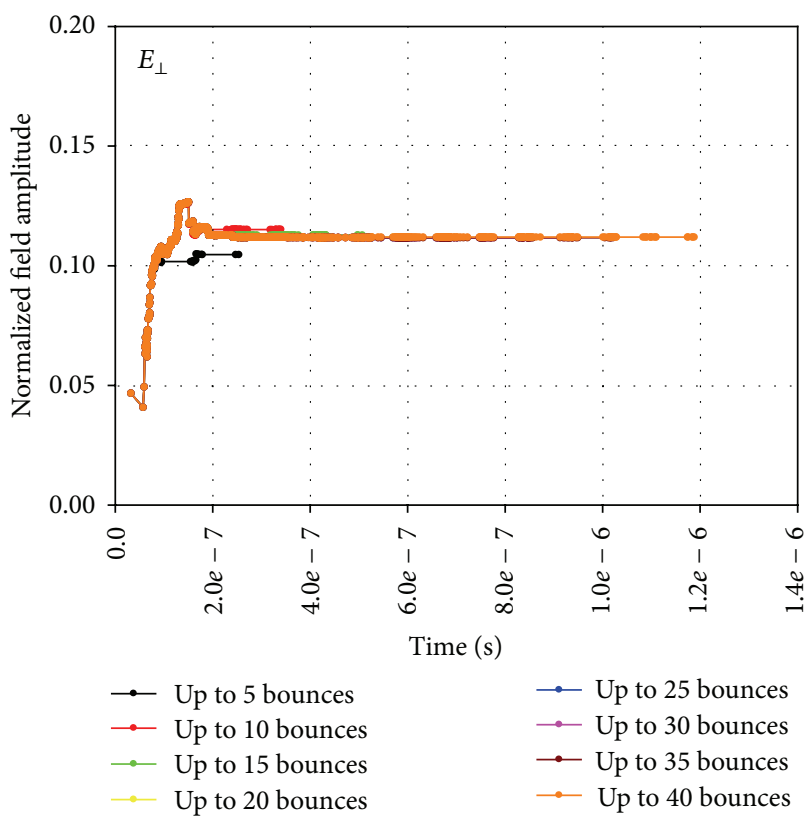

(a)
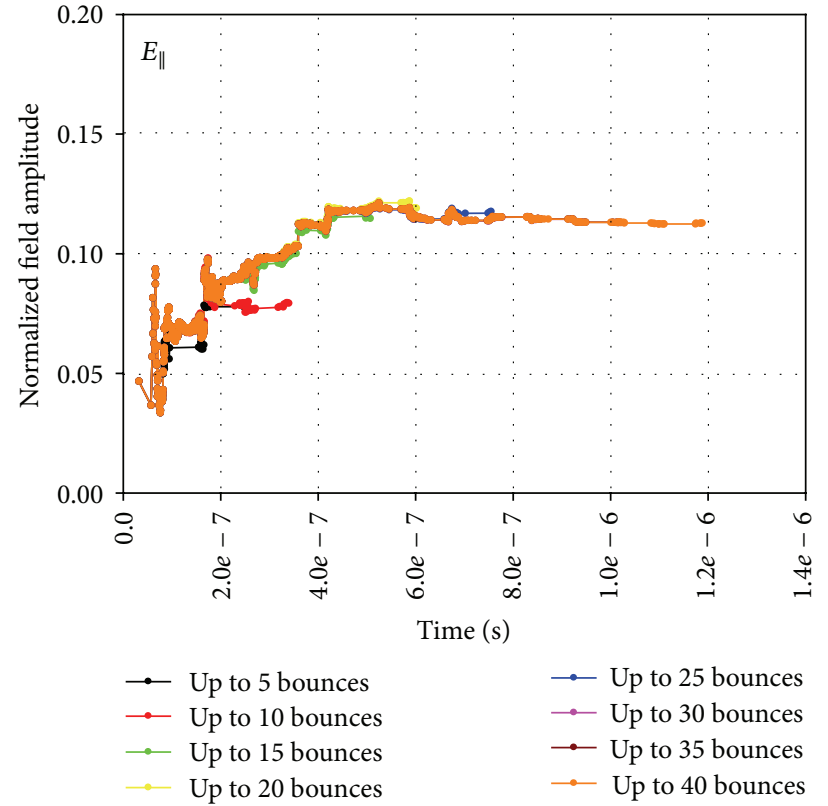

$\rightarrow$ Up to 25 bounces

- Up to 30 bounces

- Up to 35 bounces

- Up to 40 bounces

(b)

Figure 8: (a) RF field build-up inside a metal-backed dielectric $\left(\varepsilon_{r}=10-j 5 ; d=5 \mathrm{~mm}\right)$ space vehicle. Frequency $=6 \mathrm{GHz}$; polarization: perpendicular. (Though the field is normalized, the $y$-axis considered is only up to 0.20 to facilitate closer scrutiny of the plots.) (b) RF field build-up inside a metal-backed dielectric $\left(\varepsilon_{r}=10-j 5 ; d=5 \mathrm{~mm}\right)$ space vehicle. Frequency $=6 \mathrm{GHz}$; polarization: parallel. (Though the field is normalized, the $y$-axis considered is only up to 0.20 to facilitate closer scrutiny of the plots.)

The resultant normalized RF field build-up within the space vehicle for 6 and $8 \mathrm{GHz}$ is shown in Figures 8 and 9, respectively. The $y$-axis within these field normalized plots, is limited to 0.20 to facilitate a closer scrutiny. It can be readily observed that, for a metal-backed dielectric space vehicle, the ray contribution converges rapidly for both the perpendicular and parallel polarizations, both over time and the number of bounces. This is ascribed to the lossy nature of the metalbacked dielectric wall that the rays are incident upon, leading to attenuation and thus lower RF field amplitude and a rapid convergence [19].

\section{Conclusion}

The RF environment inside a space vehicle is analyzed based on the novel application of a refined ray-tracing algorithm proposed here along with hybrid surface modeling for the space vehicle. The refined ray tracing yields the required raypath data, which also facilitates visualization of all the rays that reach a particular subcube placed inside the space vehicle cabin. A ray-bunching algorithm is developed to differentiate the ray solutions as ray bunches that travel nearly parallel to reach the reception subcube. A refinement algorithm is then employed to identify the ray path within this ray bunch, which eventually converges on to the receiving point.

This algorithm generates the complete data for all the ray paths cumulatively up to a given number of bounces. In the applications where higher bounces are required, denser rays need to be launched to achieve convergence, which often leads to computational intractability. In this study, the RF field build-up at the receiving point inside a space vehicle is obtained for the rays launched at the angular separation of $0.1^{\circ}$ within the space vehicle. Again, for the class of space vehicle cabin problem analyzed in this work, for the convergence of RF field build-up, it is sufficient to consider the ray paths cumulatively up to 40 bounces, which is within the realm of computational tractability.

The RF field build-up at the receiving point within the space vehicle is determined by summing the fields associated with each ray reaching the receiver after being adjusted in phase. The effect of operational frequency on the RF field build-up is also studied. Simulations are carried out for two distinct (uplink/downlink) frequencies of 6 and $8 \mathrm{GHz}$. The results for RF field build-up are compared for a metallic space vehicle and a metal-backed dielectric space vehicle. Since the wavelength traversed by a ray over the fixed geometric path is a function of frequency, the lower the frequency is, the lesser the number of wavelengths travelled by a ray will be, leading to lesser attenuation and hence higher RF field buildup values. It is due to this fact that the total RF field build-up inside a metallic space vehicle is found to be higher at $6 \mathrm{GHz}$ as compared to $8 \mathrm{GHz}$ for both the polarizations.

The number of rays reaching the receiver increases with the ray bounce. These numerous rays incidents from different directions tend to cancel out, leaving but a net (positive/negative) RF field. Despite the large number of rays, it is only this small net RF field that contributes to the incremental rise/fall in the threshold of the RF field build-up. This leads to the slow rate of convergence. In the case of perpendicular polarization, the RF field build-up inside a metallic space 


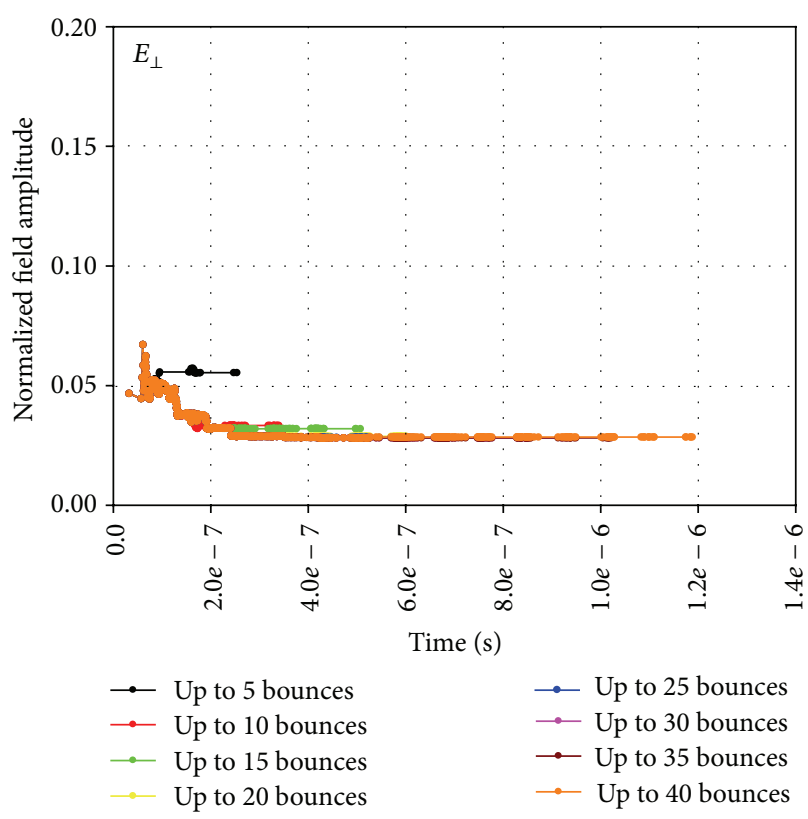

(a)
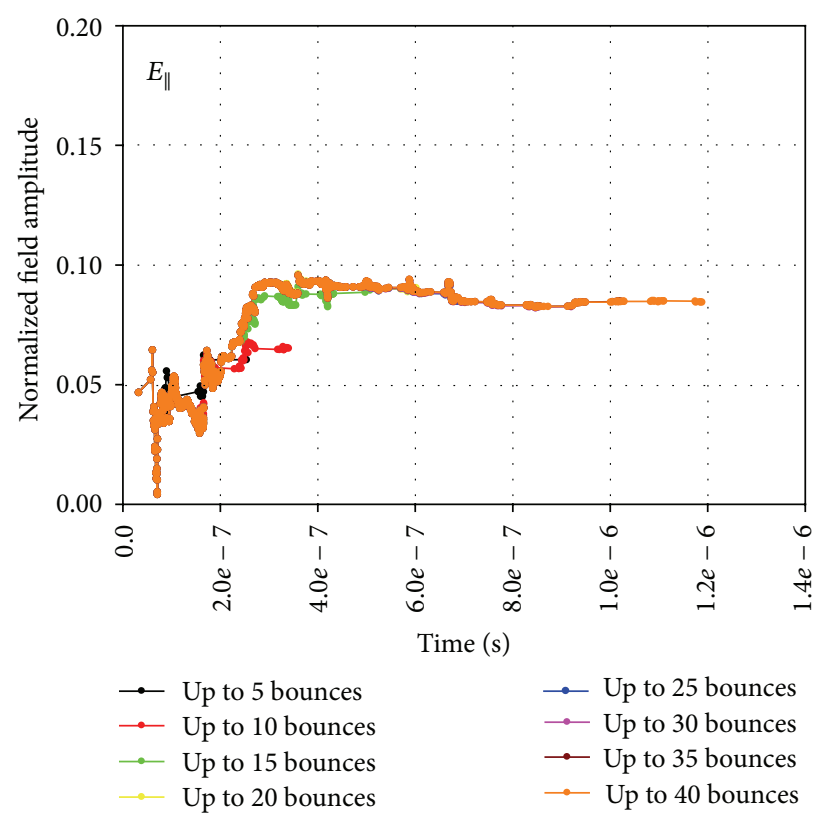

(b)

FIGURE 9: (a) RF field build-up inside a metal-backed dielectric $\left(\varepsilon_{r}=10-j 5 ; d=5 \mathrm{~mm}\right)$ space vehicle. Frequency $=8 \mathrm{GHz}$; polarization: perpendicular. (Though the field is normalized, the $y$-axis considered is only up to 0.20 to facilitate closer scrutiny of the plots.) (b) RF field build-up inside a metal-backed dielectric $\left(\varepsilon_{r}=10-j 5 ; d=5 \mathrm{~mm}\right)$ space vehicle. Frequency $=8 \mathrm{GHz}$; polarization: parallel. (Though the field is normalized, the $y$-axis considered is only up to 0.20 to facilitate closer scrutiny of the plots.)

vehicle tends to broadly increase with the number of bounces considered. This trend is on the expected lines, since it is well known that the RF field convergence is not achieved within a highly metallic reverberation chamber. In contrast, for the parallel polarization, the direction of the E-field vector changes continually after each (oblique-incidence) reflection. When the electric field associated with the multiple rays reaching the receiver is coherently superimposed, the fields with opposite phase tend to cancel each other, resulting in the rapid convergence of the total field build-up with respect to the number of bounces.

For a metal-backed dielectric space vehicle, which is the practical case under consideration, the RF field build-up converges rapidly with respect to both the elapsed time and the number of bounces. This is true for both parallel and perpendicular polarizations. This is due to the lossy nature of metal-backed dielectric wall of the space vehicle enclosure.

\section{References}

[1] J. K. Chen, G. de Veciana, and T. S. Rappaport, "Site-specific knowledge and interference measurement for improving frequency allocations in wireless networks," IEEE Transactions on Vehicular Technology, vol. 58, no. 5, pp. 2366-2377, 2009.

[2] K. H. Ng, E. K. Tameh, and A. R. Nix, "A new heuristic geometrical approach for finding non-coplanar multiple edge diffraction ray paths," IEEE Transactions on Antennas and Propagation, vol. 54, pp. 2669-2672, 2006.

[3] Y. B. Ouattara, S. Mostarshedi, E. Richalot, J. Wiart, and O. Picon, "Near- and far-field models for scattering analysis of buildings in wireless communications," IEEE Transactions on Antennas and Propagation, vol. 59, no. 11, pp. 4229-4238, 2011.

[4] H. Suzuki and A. S. Mohan, "Measurement and prediction of high spatial resolution indoor radio channel characteristic map," IEEE Transactions on Vehicular Technology, vol. 49, no. 4, pp. 1321-1333, 2000.

[5] K. W. Hurst and S. W. Ellingson, "Path loss from a transmitter inside an aircraft cabin to an exterior fuselage-mounted antenna," IEEE Transactions on Electromagnetic Compatibility, vol. 50, no. 3, pp. 504-512, 2008.

[6] N. Moraitis, P. Constantinou, F. P. Fontan, and P. Valtr, "Propagation measurements and comparison with EM techniques for in-cabin wireless networks," Eurasip Journal on Wireless Communications and Networking, vol. 2009, Article ID 784905, pp. 1-13, 2009.

[7] A. Kohmura, J. Picard, N. Yonemoto, and K. Yamamoto, "Measurement of EM field inside a cruising aircraft: potential problems for the use of mobile phones on board," Journal of Ultra-Wideband, Short Pulse Electromagnetics, vol. 9, pp. 335342, 2010.

[8] S. U. Hwu, Y. C. Loh, J. A. Dobbins, Q. D. Kroll, and C. C. Sham, "Space shuttle UHF communications performance evaluation," IEEE Aerospace and Electronic Systems Magazine, vol. 20, no. 10, pp. 9-14, 2005.

[9] R. M. Jha and W. Wiesbeck, "Geodesic constant method: a novel approach to analytical surface-ray tracing on convex conducting bodies," IEEE Antennas and Propagation Magazine, vol. 37, no. 2, pp. 28-38, 1995.

[10] P. Jackson, Ed., Jane's All the World's Aircraft 2010-2011, Jane's Information Group, Coulsdon, UK, 2010.

[11] L. Jossefsson and P. Persson, Conformal Array Antenna Theory and Design, John Wiley \& Sons, New York, NY, USA, 2006. 
[12] S. Y. Seidel and T. S. Rappaport, "Site-specific propagation prediction for wireless in-building personal communication system design," IEEE Transactions on Vehicular Technology, vol. 43, no. 4, pp. 879-891, 1994.

[13] E. Kreyszig, Advanced Engineering Mathematics, John Wiley \& Sons, New York, NY, USA, 10th edition, 2010.

[14] G. E. Athanasiadou and A. R. Nix, "A novel 3-d indoor raytracing propagation model: the path generator and evaluation of narrow-band and wide-band predictions," IEEE Transactions on Vehicular Technology, vol. 49, no. 4, pp. 1152-1168, 2000.

[15] M. Albani, G. Carluccio, and P. H. Pathak, "Uniform ray description for the PO scattering by vertices in curved surface with curvilinear edges and relatively general boundary conditions," IEEE Transactions on Antennas and Propagation, vol. 59, no. 5, pp. 1587-1596, 2011.

[16] P. Russer, Electromagnetics, Microwave Circuit and Antenna Design for Communications, Artech House, Norwood, Mass, USA, 2003.

[17] C. L. Holloway and E. F. Kuester, "Impedance-type boundary conditions for a periodic interface between a dielectric and a highly conducting medium," IEEE Transactions on Antennas and Propagation, vol. 48, no. 10, pp. 1660-1672, 2000.

[18] D.-H. Kwon, R. J. Burkholder, and P. H. Pathak, "Ray analysis of electromagnetic field build-up and quality factor of electrically large shielded enclosures," IEEE Transactions on Electromagnetic Compatibility, vol. 40, no. 1, pp. 19-26, 1998.

[19] B. Choudhury, H. Singh, J. P. Bommer, and R. M. Jha, "RF field mapping inside large passenger aircraft cabin using refined raytracing algorithm," IEEE Antennas and Propagation Magazine, vol. 55, no. 1, pp. 276-288, 2013. 

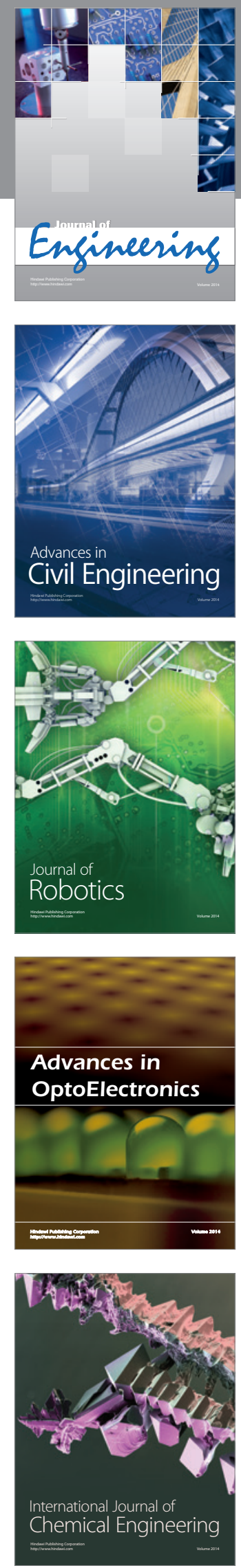

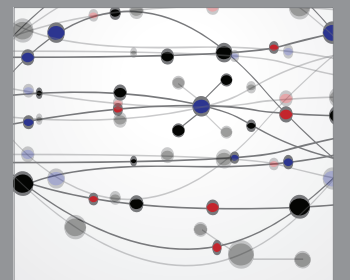

The Scientific World Journal
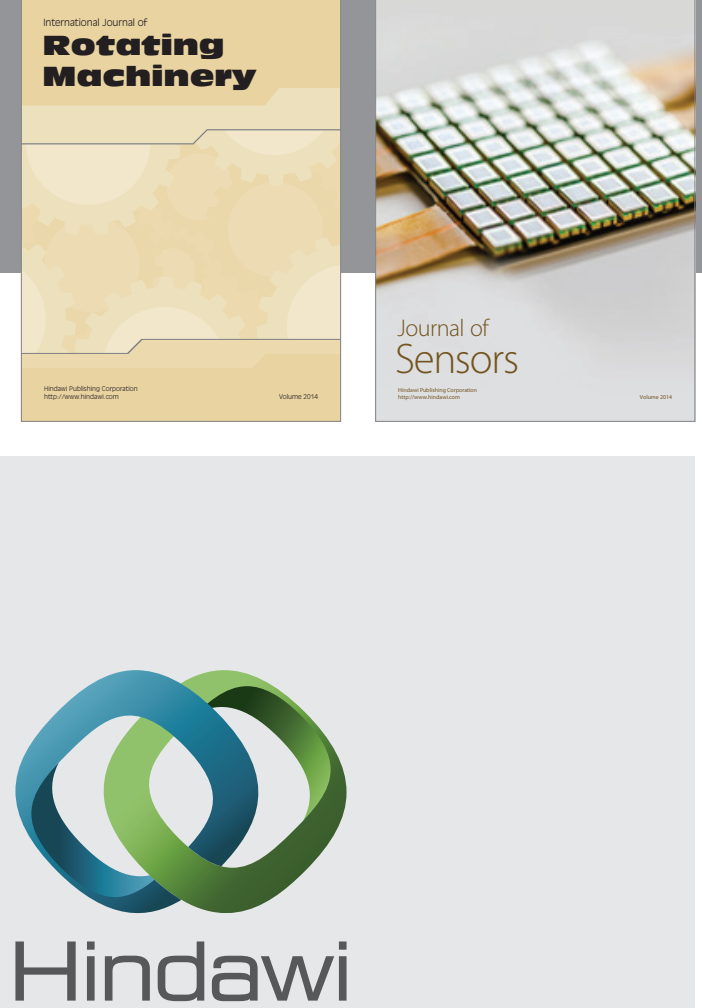

Submit your manuscripts at http://www.hindawi.com
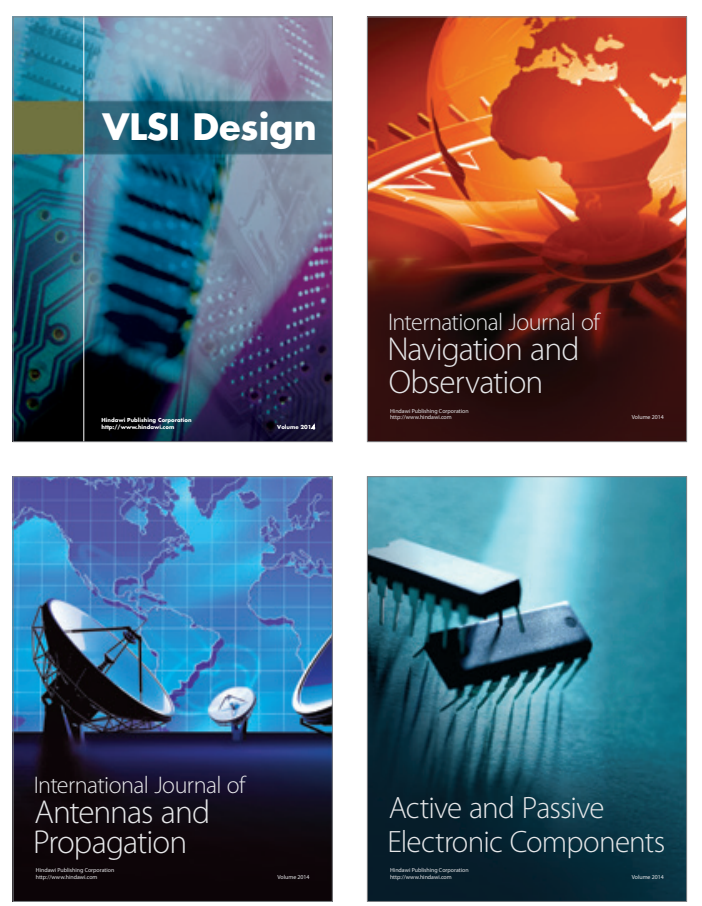
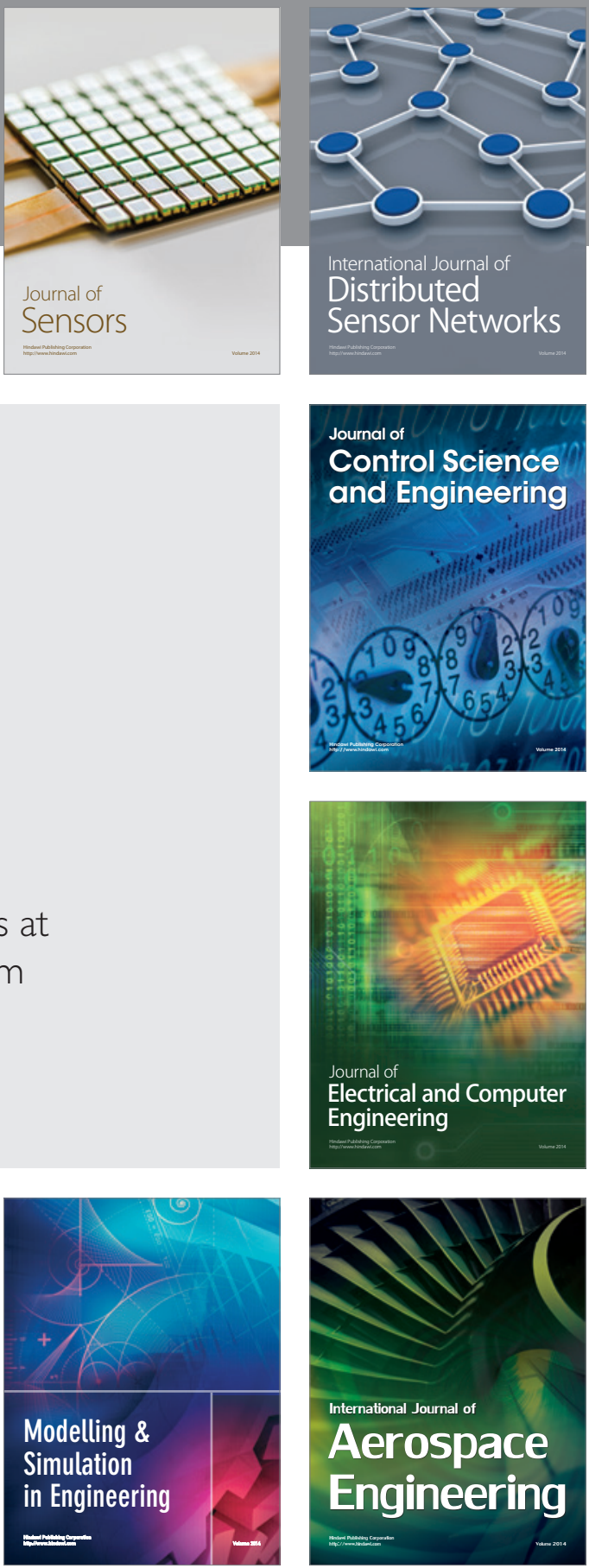

Journal of

Control Science

and Engineering
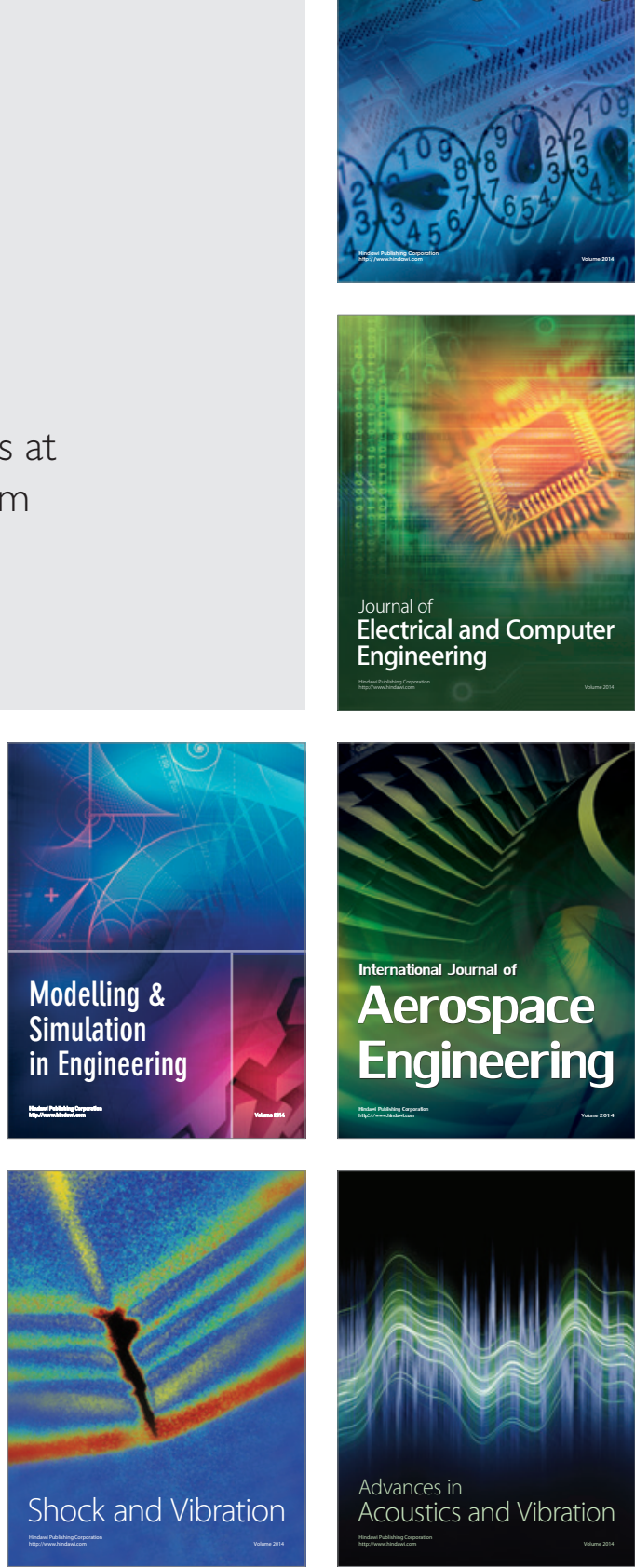